Article

\title{
Outpatient Nephrotoxic Medication Prescription after Pediatric Intensive Care Acute Kidney Injury
}

\author{
Claire Lefebvre ${ }^{1,2, *}$, Marc Dorais ${ }^{3}$, Erin Hessey ${ }^{4}$ and Michael Zappitelli ${ }^{2,5,6, *}$ \\ 1 Department of Pediatrics, Centre Hospitalier Universitaire Sainte-Justine, Montreal, QC H3T 1C5, Canada \\ 2 Department of Epidemiology, Biostatistics, and Occupational Health, McGill University, \\ Montreal, QC H3A 1A2, Canada \\ 3 StatSciences Inc., Notre-Dame-de-l'Île-Perrot, QC J7V 7P2, Canada; marc.dorais.statsciences@gmail.com \\ 4 Faculty of Medicine and Dentistry, University of Alberta, Edmonton, AB T6G 2R3, Canada; \\ hessey@ualberta.ca \\ 5 Department of Pediatrics, Division of Nephrology, Toronto Hospital for Sick Children, \\ Toronto, ON M5G 1X8, Canada \\ 6 Department of Pediatrics, Division of Nephrology, McGill University, Montreal, QC H3A 1A2, Canada \\ * Correspondence: claire.lefebvre@umontreal.ca (C.L.); michael.zappitelli@sickkids.ca (M.Z.); \\ Tel.: +1-514-345-4931 (ext. 2770) (C.L.)
}

check for updates

Citation: Lefebvre, C.; Dorais, M.; Hessey, E.; Zappitelli, M. Outpatient Nephrotoxic Medication Prescription after Pediatric Intensive Care Acute Kidney Injury. Children 2021, 8, 948. https://doi.org/10.3390/

children 8110948

Academic Editor: Stella Stabouli

Received: 9 September 2021

Accepted: 6 October 2021

Published: 21 October 2021

Publisher's Note: MDPI stays neutral with regard to jurisdictional claims in published maps and institutional affiliations.

Copyright: (c) 2021 by the authors. Licensee MDPI, Basel, Switzerland. This article is an open access article distributed under the terms and conditions of the Creative Commons Attribution (CC BY) license (https:// creativecommons.org/licenses/by/ $4.0 /)$.

\begin{abstract}
Background: Nephrotoxic medication (NTM) avoidance may prevent further kidney damage in children with acute kidney injury (AKI). We compared outpatient NTM prescriptions in children with or without AKI during pediatric intensive care (PICU) hospitalization. We hypothesize that children with AKI are prescribed NTMs at the same rate as those without it. Methods: This was a retrospective administrative data study of children $<18$ years, admitted to two PICUs in Montreal, Canada, from 2003 to 2005, with $\geq 30$ days of provincial drug coverage. We evaluated the presence of $\geq 3$ outpatient NTM prescriptions during the first year and 5 years after PICU discharge. Results: Of 970 children, $23 \%$ had PICU AKI. In the 1st-5th years after discharge, 18\% AKI vs. 10\% non-AKI and $13 \%$ AKI vs. $4 \%$ non-AKI patients received $\geq 3$ NTM prescriptions, respectively. There was no association between PICU AKI and prescription of $\geq 3$ NTMs during the first year (adjusted RR 1.02 [95\% CI 0.95-1.10]) nor in the first 5 years post-discharge (adjusted RR 1.04 [95\%CI 0.96-1.12]). Conclusions: By offering a better understanding of the current state of outpatient NTM prescription to children with AKI, our study is a step toward considering strategies such as knowledge translation interventions for decreasing NTM exposure and improving outcomes in children with AKI.
\end{abstract}

Keywords: pediatrics; renal; kidney disease; nephrotoxicity; prescription patterns

\section{Introduction}

Acute kidney injury (AKI) is an important public health concern. AKI or abrupt kidney dysfunction occurs in $\sim 20 \%$ of children in the pediatric intensive care unit (PICU) and $<5 \%$ of all hospitalized children [1]. While its etiology is commonly multifactorial in the hospital setting, AKI can occur and be exacerbated due to administration of nephrotoxic medications [2-4]. There is mounting evidence that AKI may be associated with the development of subsequent chronic kidney disease (CKD) [5,6], which is a potent cardiovascular disease risk factor $[7,8]$.

There are no standardized guidelines on how to follow children with AKI after hospital discharge. Recent work suggests that many children with AKI are not followed for the possible development of CKD, suggesting that primary care providers may not be aware of the potential long-term effects of AKI [9]. An important concept in managing AKI and $\mathrm{CKD}$ is preventing further kidney damage, including nephrotoxic medication avoidance. If AKI is a risk factor for long-term CKD development, ideally, children with a history of AKI would not receive nephrotoxic medications, unless necessary. Nephrotoxic medication 
avoidance after AKI represents an opportunity for process of care and education interventions to mitigate the effects of AKI on long-term kidney function. We recently showed in a large UK cohort that children with CKD are often prescribed nephrotoxic medication by primary care providers [10]. Whether healthcare providers alter nephrotoxic medication prescriptions based on the history of AKI in children remains completely unknown.

Using administrative healthcare data, our objective was to describe and compare outpatient prescriptions of nephrotoxic medications after a PICU hospitalization in children who did vs. children who did not develop AKI during PICU admission. We hypothesized that due to the under-recognition of the potential importance of AKI in long-term kidney outcomes, children who developed AKI during PICU admission would be prescribed nephrotoxic medications after discharge at a rate similar to children who did not develop AKI.

\section{Materials and Methods}

\subsection{Design, Setting, and Patient Selection}

This is a secondary analysis of a previously published retrospective cohort study including children $<18$ years old with a valid provincial health card number, who were admitted to the PICU of the Montreal Children's Hospital (MCH) (Montreal, Quebec, Canada) or the Centre Hospitalier Universitaire (CHU) Sainte-Justine (Montreal, Quebec, Canada) between 1 January 2003 and 31 March 2005, inclusively. Children with end-stage kidney disease (ESKD, including kidney transplant) before the index admission were excluded [11]. For this analysis, only the following children were included: survived to the index hospital discharge; registered to the Régie de l'assurance maladie du Québec (RAMQ, described below) provincial drug plan for at least 30 days after index hospitalization discharge. We only included the first PICU-associated hospitalization during the study period. (Figure 1). The follow-up start date was the date of discharge from the index hospitalization, and follow-up continued for up to 7 years after hospital discharge, death, or patient's exit from the provincial drug plan for $\geq 6$ months. Approvals from institutional research ethics boards and the Commission d'Accès à l'Information du Québec (provincial data monitoring board) were obtained.

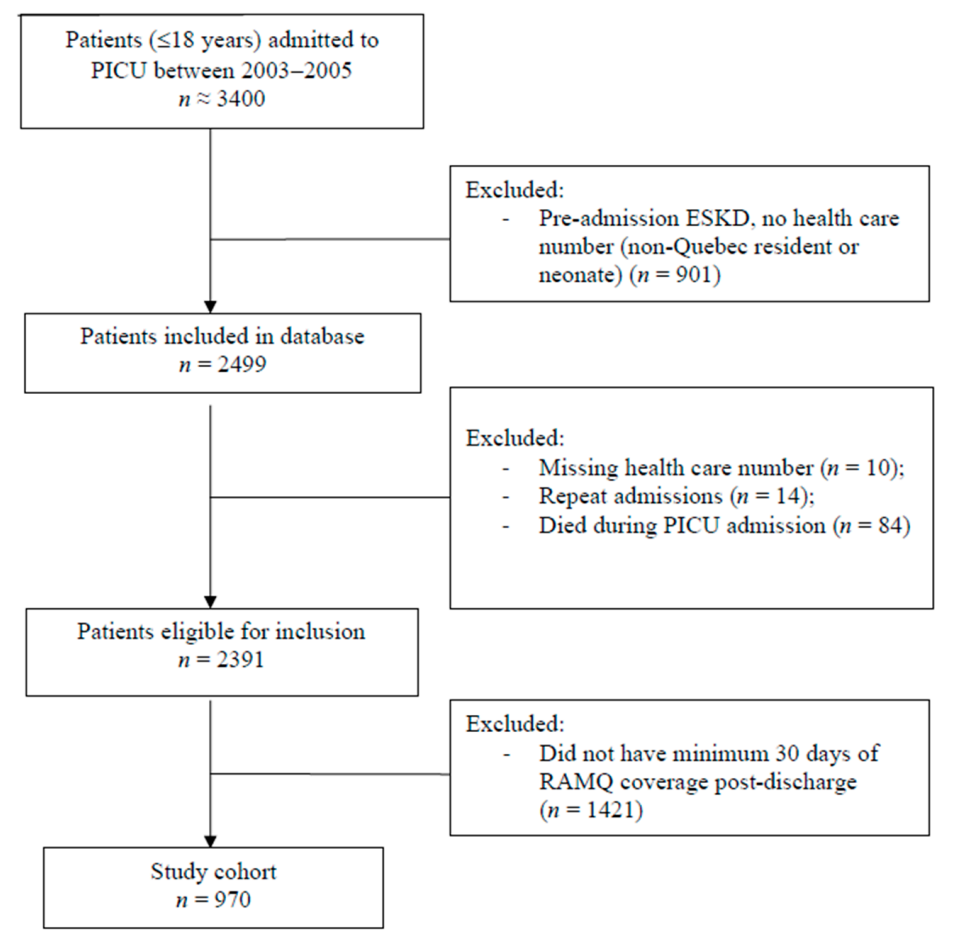

Figure 1. Flow Diagram of Patient Inclusion from the PICU and RAMQ Database. Abbreviations: PICU: pediatric intensive care unit; ESKD: end-stage kidney disease; RAMQ: Régie de l'assurance maladie du Québec. 


\subsection{Data Collection and Sources}

Index hospitalization and PICU data were collected by a retrospective chart review on standardized forms which were pre-tested for reliability [12]. These variables, previously described, included: sociodemographic data; pre-PICU patient characteristics (comorbidities, baseline kidney function); PICU admission variables (e.g., primary diagnosis; Pediatric Risk of Mortality (PRISM) score [13]; daily medication (including vasopressors, diuretics, antibiotics, non-steroidal anti-inflammatories; steroids), duration of mechanical ventilation, daily serum creatinine (SCr), and urine output); and hospital outcomes (e.g., length of stay).

Chart data were merged with data from the Quebec Vital Statistics Registry (to evaluate post-discharge mortality) and administrative healthcare databases of the RAMQ and MedEcho (collectively including administrative healthcare data from 1 year before the first PICU admission in the cohort during the study period to 5 years after the last admission during the study period) [11]. The administrative healthcare databases provided data on healthcare encounters (e.g., hospitalizations, physician visits), demographic and diagnosis/procedure codes (using the International Classification of Diseases-9 and 10) data (used to calculate the social and material deprivation indices as a marker of socio-economic status as well as the Pediatric Complexity Algorithm to reflect patient health complexity) [14,15]. Outpatient medication prescription data are included in the RAMQ databases.

Quebec has a single-payer health insurance plan. However, medications are reimbursed by a separate public drug plan which covers $\sim 48 \%$ of the province's children [16]. The RAMQ pharmaceutical services file provides information on medications reimbursed by the provincial insurer, including drug name, date the prescription was collected, and prescription dose, form, and duration. For children $<18$ years of age to be covered, their parents/guardians must be registered with the RAMQ drug plan. Patients are registered to the drug plan until they move out of a province or enroll in a private insurance plan (e.g., employer). We defined "continuous RAMQ medication coverage" as gaps in coverage $<6$ months.

\subsection{AKI Definition}

AKI during PICU hospitalization was defined from retrospective chart data using KDIGO (Kidney Disease: Improving Global Outcomes) criteria, classifying AKI based on the degree of SCr rise from baseline or the urine output decrease [17]. For patients with SCr measured in the three months before index PICU admission, the lowest $\mathrm{SCr}$ was the baseline SCr [17]. For patients with no baseline SCr available, we estimated it using the Chronic Kidney Disease in Children (CKiD) bedside equation (eGFR $=36.5 \times$ (height in $\mathrm{cm} / \mathrm{SCr}$ in $\mu \mathrm{mol} / \mathrm{L}$ )) (1) to back-calculate baseline $\mathrm{SCr}$, using patient height and assuming an age-based normative estimated glomerular filtration (eGFR) value (2) (for children $\leq 2$ years) or an eGFR $=120 \mathrm{~mL} / \mathrm{min} / 1.73 \mathrm{~m}^{2}$ (for children $>2$ years) $[18,19]$. For patients with no height available, we used the height-independent Hoste equation [20] to back-calculate baseline SCr. Both equations have been validated in our population [21]. Patients for whom AKI could not be ascertained, because no SCr or urine output was recorded during PICU stay, were assumed not to have AKI [22]. The rationale behind this is that children without $\mathrm{SCr}$ or urine output measured during PICU are unlikely to be severely ill and therefore unlikely to have developed AKI. We previously showed that patients for whom AKI ascertainment data were not available were extremely similar in characteristics to patients with no AKI [23]. As a secondary analysis, we evaluated the presence of Stage 2 AKI or worse ( $\geq$ doubling of $\mathrm{SCr}$ from baseline or dialysis for AKI) during PICU (vs. not having AKI or Stage 1 AKI).

\subsection{Outcome: Post-Discharge Outpatient Medication Prescriptions}

Our definition of a nephrotoxic medication was derived from three recent studies using robust methodologies to generate lists of medications recognized as nephrotoxic in adults or children [24-26]. In addition to considering data from these studies, we assessed the face validity of the included medications. We reached consensus about 
the inclusion of additional drugs that did not appear on these lists but were deemed to have sufficient evidence supporting their nephrotoxicity. We then developed two lists: one of established nephrotoxic medications and another of potential nephrotoxic medications (Supplementary Table S1). Established nephrotoxic medications included those considered nephrotoxic in at least two of the three reference studies. To this list, we added medications from the following classes: non-steroidal anti-inflammatory drugs (NSAIDs), aminoglycoside antibiotics, proton pump inhibitors, and salicylates. Our second list was broadened to include medications considered "potentially" nephrotoxic (providing as wide a capture of potential nephrotoxic medications as possible). This list included our established nephrotoxic medications plus medications considered to have nephrotoxic "potential" in any of the three reference studies or medications which only one of the three studies considered established nephrotoxic. Because of their possible indication in kidney disease following AKI, we considered angiotensin-converting enzyme (ACE) inhibitors to be potentially nephrotoxic in our study.

Our primary outcomes were: (1) presence (yes/no) of $\geq 3$ established nephrotoxic medication prescriptions during the first year and during the first 5 years after PICU discharge and (2) receiving (yes/no) $\geq 3$ established nephrotoxic medication prescriptions at 1-year intervals after PICU discharge. The rationale for using a cutoff of $\geq 3$ medications was the association between this level of nephrotoxic medication exposure and incident AKI in hospitalized children [3,25]. Results were repeated for established and potential nephrotoxic medications.

Secondary outcomes included (a) presence of $\geq 3$ potential nephrotoxic medication prescriptions and (b) presence of $\geq 3$ established and potential nephrotoxic medication prescriptions from different classes (e.g., NSAIDS; antivirals). The secondary outcomes were evaluated during the first year and the first 5 years of follow-up.

\subsection{Analysis}

Analyses were performed using R, version 3.3.3 (R Foundation for Statistical Computing, Vienna, Austria) and SAS statistical software, release 9.4 (SAS Institute Inc., Cary, NC, USA). Characteristics were reported as appropriate based on distribution, and variables were compared between groups using distribution-appropriate univariable analyses (e.g., t-tests, Mann-Whitney U tests, Chi-square with or without Yate's correction, Fisher's exact test). Proportions of AKI vs. non-AKI patients who received $\geq 3$ individual established nephrotoxic medication prescriptions after hospital discharge and at 1-year intervals, up to 5 years after discharge, were compared. Multivariable log binomial regression was used to calculate risk ratios (RR, 95\% confidence interval [CI]) for the association between AKI (and also Stage 2/3 AKI) with receiving $\geq 3$ established nephrotoxic medication prescriptions during the first year and in the first 5 years of follow-up. Similar analyses were performed to evaluate associations with our secondary outcomes (i.e., number of potential nephrotoxic medication prescriptions). The proportions of the most common classes of nephrotoxic medications prescribed by AKI-status group were calculated.

A priori covariates included in all multivariable models were age, gender, rural vs. urban home, pediatric medical complexity [15], and social and material deprivation indices [14]. For each multivariable model of our primary and secondary outcomes, additional covariates were selected if they had a significant association $(p<0.05)$ with both the exposure (AKI) and the specific outcome in univariable analyses. If data were missing for covariates, a complete case approach was used (i.e., patients with data on all relevant covariates were included in multivariable regressions. This resulted in $<6 \%$ loss of subjects in all multivariable analyses). If there were too few observations within categorizations of a covariate, the covariate was excluded from the model. Cardiac surgery was explored as an effect modifier (planned a priori analysis) by including it as an interaction term with AKI in our multivariable model for our primary outcome ( $\geq 3$ established nephrotoxic medication prescriptions in the first year of follow-up). In regression models evaluating outcomes at 
1 and 5 years of follow-up, only patients with ongoing follow-up at those time intervals were included in the analyses.

\section{Results}

\subsection{Cohort Description}

There were 2499 patients hospitalized at the PICU from 2003 to 2005 . Figure 1 shows reasons for exclusion, leading to an analysis cohort of 970 children (the majority excluded due to neonatal age and lack of RAMQ provincial drug plan coverage for $\geq 30$ days). RAMQ drug plan coverage in our cohort closely resembled province-wide RAMQ coverage rates of $48 \%$ for children [16]. The characteristics of included and excluded children were similar, except that a higher proportion of the included children had elevated levels of social and material deprivation (Supplementary Table S2). Two hundred and twenty-three children (23\%) developed AKI during PICU hospitalization ( $n=85$ (9\%) with $\geq$ Stage 2 AKI). Comparisons of characteristics between AKI and non-AKI patients are presented in Supplementary Table S3. Average follow-up time was 5.9 years. At 1 and 5 years of follow-up, 953 (98\%) and 910 (94\%) patients (no statistically significant difference between AKI groups), respectively, had continuous follow-up, defined as follow-up with less than a 6-month gap in RAMQ drug coverage.

\subsection{Association of AKI with the Number of Nephrotoxic Medication Prescriptions}

Figure 2 illustrates the proportions of patients with and without AKI who received $\geq 3$ established and potential nephrotoxic medication prescriptions at yearly intervals after hospital discharge. During the first year, $18 \%$ of AKI and $10 \%$ of non-AKI patients received $\geq 3$ established nephrotoxic medication prescriptions. In addition, 39\% of AKI and $29 \%$ of non-AKI patients received $\geq 3$ potential nephrotoxic medication prescriptions $(p=0.02)$. In the fifth year post-discharge, $13 \%$ of AKI and $4 \%$ of non-AKI patients received $\geq 3$ established nephrotoxic medication prescriptions, whereas $22 \%$ of AKI and $12 \%$ of non-AKI patients received $\geq 3$ potential nephrotoxic medication prescriptions $(p<0.01)$. When considering the entire 7 years of follow-up, $59 \%$ of AKI and $56 \%$ of non-AKI patients received $\geq 3$ potential nephrotoxic medication prescriptions at some time $(p=0.58)$.

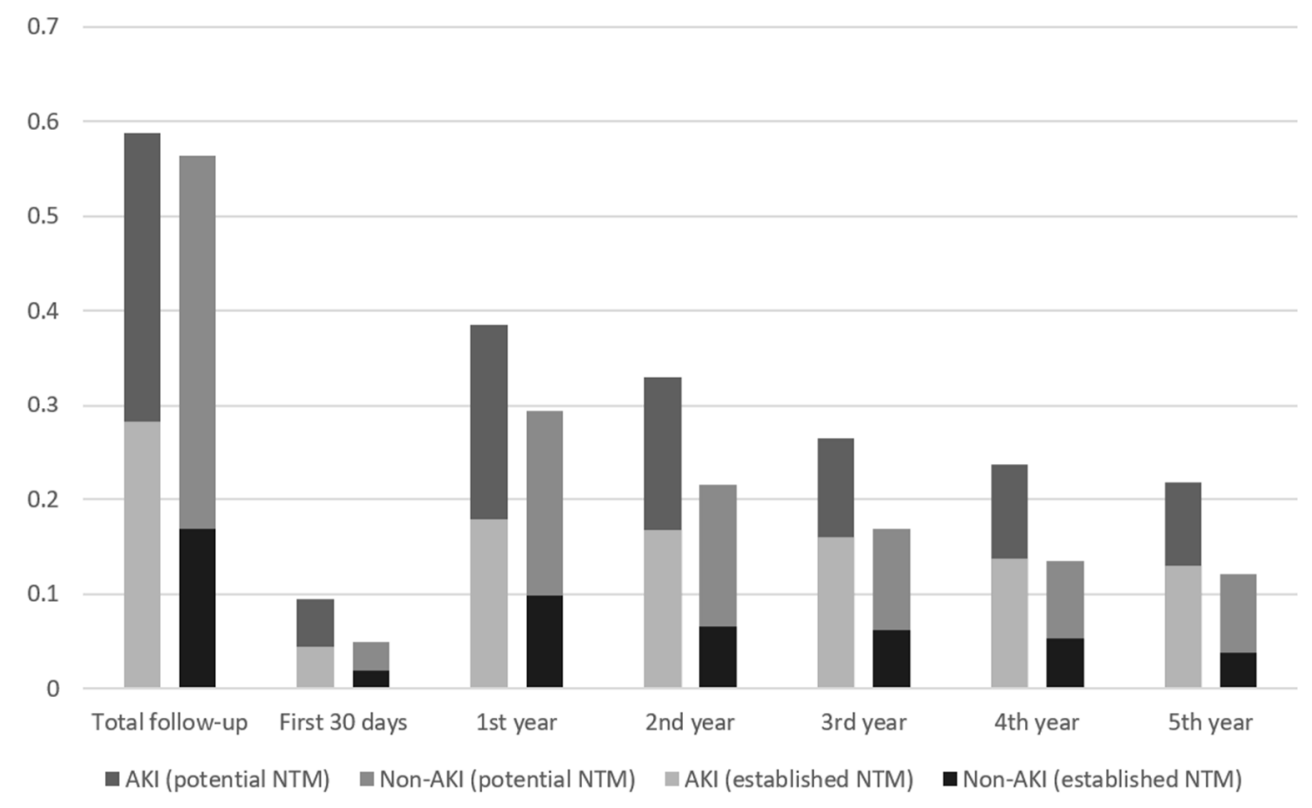

Figure 2. Percentages of AKI and non-AKI Patients Receiving $\geq 3$ Nephrotoxic Medication Prescriptions by Year Since PICU Discharge. Bar chart of proportions of AKI and. Non-AKI patients who received $\geq 3$ nephrotoxic medication prescriptions by year since PICU discharge. Numbers above figure bars represent the total number of participants with continuous follow-up at the time interval after discharge (denominator). 
Table 1 shows that there was no association between AKI during PICU admission and prescription of $\geq 3$ established nephrotoxic medications during the first year after hospital discharge (adjusted RR 1.02 [95\% CI 0.95-1. 10], adjusted for variables shown in Table 1 legend) or in the 5 years after hospital discharge (adjusted RR 1.04 [95\%CI 0.96-1.12], adjusted for variables shown in Table 1 legend). The presence of $\geq$ Stage 2 AKI (vs. no AKI or Stage $1 \mathrm{AKI}$ ) was also not associated with the outcomes in adjusted analyses (Table 1).

Table 1. Adjusted Association Between AKI and Administration of $\geq 3$ Established Nephrotoxic Medication Prescriptions and Classes.

\begin{tabular}{|c|c|c|c|c|}
\hline & \multicolumn{2}{|c|}{ AKI vs. no AKI } & \multicolumn{2}{|c|}{ Stage $2 / 3$ vs. no AKI or Stage 1 AKI } \\
\hline & $\begin{array}{c}\text { First Year } \\
(n=953) \\
\text { Adjusted RR } \\
(95 \% \text { CI })\end{array}$ & $\begin{array}{c}\text { First } 5 \text { Years } \\
(n=910) \\
\text { Adjusted RR } \\
(95 \% \text { CI })\end{array}$ & $\begin{array}{c}\text { First Year } \\
(n=953) \\
\text { Adjusted RR } \\
(95 \% \text { CI })\end{array}$ & $\begin{array}{c}\text { First } 5 \text { Years } \\
(n=910) \\
\text { Adjusted RR } \\
(95 \% \text { CI })\end{array}$ \\
\hline $\begin{array}{l}\geq 3 \text { established nephrotoxic } \\
\text { medication prescriptions }\end{array}$ & $1.02(0.95-1.10)^{\mathrm{a}}$ & $1.04(0.96-1.12)^{b}$ & $1.03^{\mathrm{a}}(0.92-1.14)$ & $1.04(0.94-1.16)^{b}$ \\
\hline $\begin{array}{c}\geq 3 \text { established nephrotoxic } \\
\text { medication classes }\end{array}$ & $1.02(0.97-1.08)$ & $1.02(0.96-1.09)^{c}$ & $1.02(0.91-1.15)$ & $1.02^{\mathrm{c}}(0.93-1.13)$ \\
\hline
\end{tabular}

Abbreviations: AKI: Acute Kidney Injury; RR: Risk Ratio. NB: All models include covariates: age, gender, rural versus urban home address, pediatric medical complexity algorithm, and social and material deprivation indices. Additional model covariates (associated with the exposure and outcome of the analysis) include: a cardiac surgery, trauma, diabetes, diuretics, antibiotics, PRISM score; b cardiac surgery, trauma, diuretics, antibiotics, PRISM score; c antibiotics.

Cardiac surgery was not an effect modifier of the AKI-outcome relation (i.e., did not alter the association between AKI and nephrotoxic medication prescription; interaction term $p$ value $=0.4$ ). In the 1 - and 5-year outcome multivariable analyses, higher pediatric medical complexity was consistently associated with the presence of $\geq 3$ established nephrotoxic medication prescriptions ( $p<0.05$ in adjusted models). When we considered potential nephrotoxic medications in our definition, adjusted associations between AKI in PICU with 1-year and 5-year presence of $\geq 3$ potential nephrotoxic medication prescriptions were extremely similar (shown in Supplementary Table S4).

\subsection{Association of AKI with the Number of Nephrotoxic Prescriptions from Different Classes}

Figure 3 shows that $3 \%$ of AKI and $<1 \%$ of non-AKI children received medications from $\geq 3$ separate established nephrotoxic classes in 1 year after hospital discharge $(p<0.01)$; in the fifth year after discharge, no patient received medications from $\geq 3$ potential nephrotoxic classes. Table 1 shows that there was no statistically significant association between AKI and prescription of $\geq 3$ established nephrotoxic medication prescriptions from different classes (adjusted RR 1.02 [95\% CI 0.97-1.08] in the first year, adjusted RR 1.02 [95\% CI 0.96-1.09] in the first five years; bottom of Table 1, adjusted for variables indicated in the legend). These results were extremely similar when defining the exposure as the presence of $\geq$ Stage 2 AKI (shown in Table 1).

\subsection{Distribution of the Prescribed Nephrotoxic Medication Classes}

When considering the full 7 years of available follow-up, the most commonly prescribed established or potential nephrotoxic medication class was beta-lactam antibiotics ( $23 \%$ of patients with AKI and $42 \%$ of patients without AKI) (Supplementary Tables S5 and S6, respectively). ACE-inhibitors were the second most commonly prescribed medication class to patients with AKI ( $18 \%$ of AKI patients throughout the study period). Proton pump inhibitors represented another commonly prescribed medication class and were prescribed to $14 \%$ and $13 \%$ of AKI and non-AKI patients, respectively. NSAIDs were prescribed to 3\% of all patients, regardless of their AKI status. 


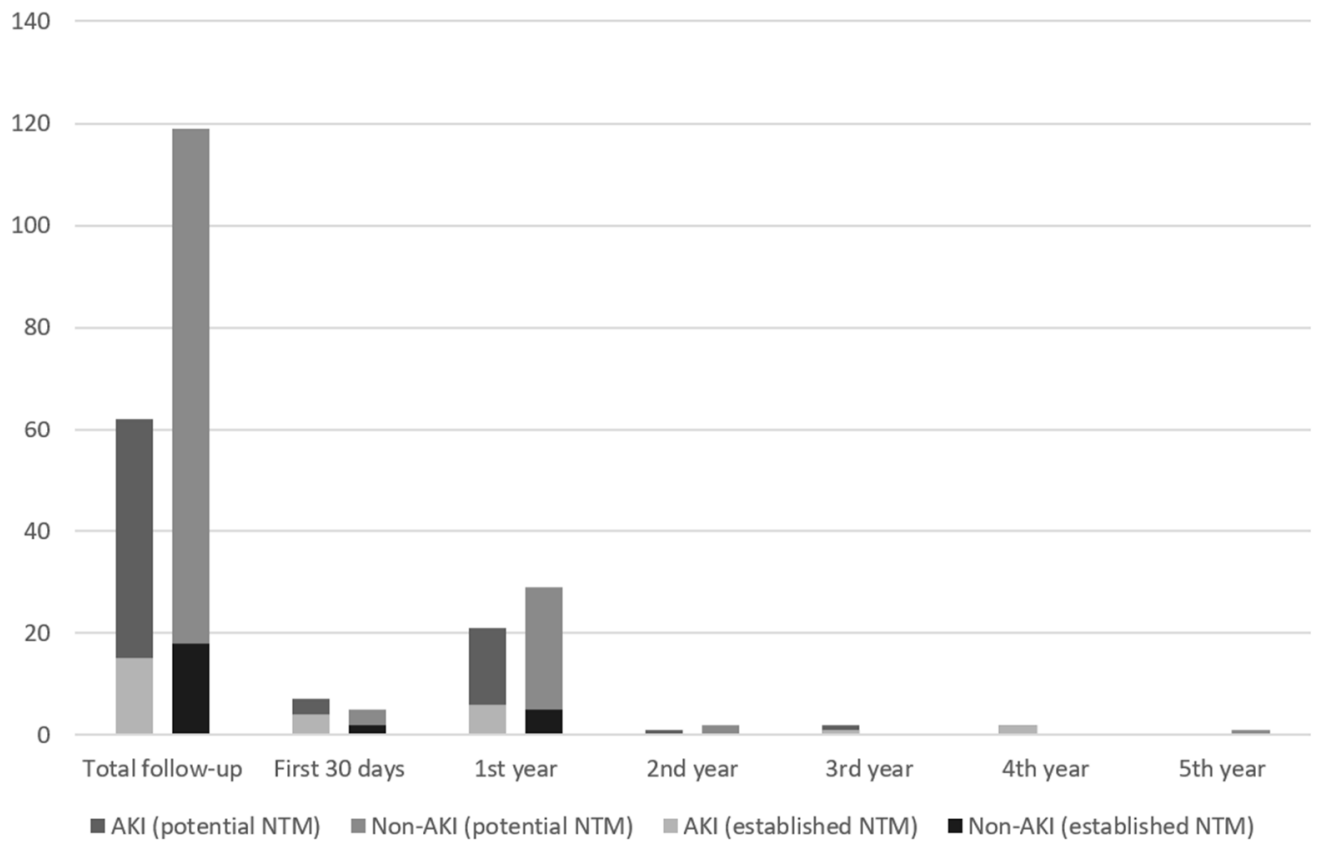

Figure 3. Percentages of AKI and non-AKI Patients Receiving $\geq 3$ Nephrotoxic Medication Classes by Year Since PICU Discharge. Bar chart of proportions of AKI and Non-AKI patients who received $\geq 3$ nephrotoxic medication prescriptions by year since PICU discharge. Numbers above bars represent the total number of participants with continuous follow-up at the time interval after discharge (denominator).

\section{Discussion}

In the first year after PICU discharge, the RR of receiving $\geq 3$ nephrotoxic medication prescriptions for children with AKI did not differ substantially from that for children without AKI. Almost $20 \%$ of the patients with a documented episode of AKI were prescribed $\geq 3$ established nephrotoxic medication prescriptions in the year after their discharge from the PICU compared to $10 \%$ of children without AKI. Three or more potential nephrotoxic medications were prescribed to just under $40 \%$ of children with AKI and to just under 30\% of children without AKI in the year following PICU discharge.

These findings are important because children with AKI may be at risk for subsequent AKI and for CKD progression [5,27]. Avoiding nephrotoxic medications is important in this population, as it represents one of the few modifiable risk factors for kidney disease progression. Though individual nephrotoxic medications are sometimes inconsistently associated with AKI, in the pediatric non-critical care population, exposure to $\geq 3$ nephrotoxic medications has been shown to nearly double the positive predictive value of developing AKI (compared to $\leq 2$ nephrotoxic medications) and has been previously reported to be associated with a $30 \%$ AKI incidence [3,25]. The high burden of outpatient nephrotoxic medication exposure in our study suggests a need for increased awareness of this practice among discharging and treating physicians. A previous study examining healthcare utilization in our cohort's patients noted that fewer than $25 \%$ of those patients with a PICU diagnosis of AKI saw a nephrologist in the 5 years after their PICU discharge [22]. This highlights the need for post-AKI follow-up guidelines for these children.

To our knowledge, this is the first study evaluating the prescription of outpatient nephrotoxic medication following AKI in a PICU population. Most studies in this population have focused on inpatient prescriptions. Existing hospital studies have exposed worrying trends in prescribing practices to children with AKI, as well as promising interventions to mitigate these trends. One study described the effects of an institution-wide $\mathrm{SCr}$ surveillance program which resulted in a dramatic drop in inappropriate prescriptions and in cases of AKI (38\% and 64\%, respectively) [28]. Hospital-based studies overlook an important aspect of the clinical care pathway of many AKI patients-the outpatient setting. 
This is often the most frequent point of contact these children have with health care and has inherent differences with respect to the hospital setting. Regular SCr surveillance is feasible in hospital and unlikely to result in an increased burden of care in children already undergoing regular blood tests; such interventions may be impractical in the outpatient setting. Educational campaigns focused on increasing awareness of the importance of nephrotoxic medication avoidance may be of value.

Several commonly prescribed nephrotoxic medications identified were medications known to be commonly used in children (e.g., beta-lactam antibiotics, proton-pump inhibitors) and may often be difficult to avoid. However, alternatives do exist, and we propose, as supported by our study, that in the context of avoiding the overuse of antibiotic prescriptions, considering potential adverse effects should play a role in decision making. ACE-inhibitors were commonly prescribed to patients with a history of AKI. This may reflect the high proportion of cardiac surgery patients for whom these medications may be indicated for afterload reduction and for the treatment of hypertension. However, considering their nephrotoxic potential, their widespread use in these at-risk populations should prompt the consideration of a close monitoring of kidney function.

Our study has limitations. This study was conducted in a region with universal health care, and the results may therefore not be applicable to other contexts. Our hospitalization data also dates to 2003-2005, and prescribing practices may have changed since then. We did not have the indication for nephrotoxic medication prescriptions and can therefore not comment directly on the justification for their use or whether safer alternatives could reasonably have been considered. Though our study details evidence of a high nephrotoxic burden in children with previous AKI, we cannot comment on the impact of prescribing nephrotoxic medication on the evolution of these children's kidney disease or on AKI recurrence. Furthermore, the absence of SCr measurements in a large proportion of our cohort made the identification of AKI difficult in many patients. This is a common feature of many studies of pediatric AKI and highlights how important it is to record these parameters in all children admitted to the PICU. However, our decision to include urine output criteria as an inclusion parameter would have helped to offset this lack of sensitivity. Lastly, in our study, the proportion of patients exposed to $\geq 3$ potential or established nephrotoxic medications decreased with each passing year after PICU discharge, which suggests that the initial prescriptions may have been a response to the acute illness leading to their PICU hospitalization. We attempted to account for disease severity in our models by controlling for pre-admission diagnoses and PICU factors, but there may have been residual confounding.

\section{Conclusions}

By offering a better understanding of the current state of nephrotoxic medication prescribing to children with AKI in the outpatient setting, our study is a step toward considering strategies such as knowledge translation interventions for decreasing nephrotoxic medication exposure and, ultimately, improving outcomes in children with AKI.

Supplementary Materials: The following are available online at https:/ / www.mdpi.com/article/10 $.3390 /$ children8110948/s1. Table S1: List of Nephrotoxic Medications Included in the Study with RAMQ (provincial drug plan coverage) Code, Drug Name, Pharmacologic Category, and Status as Established or Potential Nephrotoxic Medication, Table S2: Comparisons between included and excluded children, Table S3: Patient characteristics by AKI status and by outcome status during the whole follow-up, Table S4: Adjusted Association Between AKI and Receiving $\geq 3$ Potential Nephrotoxic Medication Prescriptions and Classes, Table S5: Dispensed medications for children with AKI, Table S6: Dispensed medications for children with no AKI.

Author Contributions: Conceptualization, C.L. and M.Z.; Data curation, M.Z.; Formal Analysis, C.L. and M.D.; Funding acquisition, C.L. and M.Z. Investigation, M.Z.; Methodology, M.Z.; Project administration, M.Z.; Resources, M.Z.; Software, M.D.; Supervision, M.Z.; Validation, M.Z.; Visualization, C.L.; Writing—original draft, C.L. and M.Z.; Writing—review \& editing, C.L., M.D., E.H. and M.Z. All authors have read and agreed to the published version of the manuscript. 
Funding: An operating grant to MZ from the Fonds de Recherche du Québec-Santé (grant number 22888) was provided to perform the original data collection for this study. MZ utilized unrestricted research operating funds from the Toronto Hospital for Sick Children and the SickKids Research Institute to complete the analysis of this work. CL received a research trainee salary award from the Canadian Institutes for Health Research and from the Fonds de recherche du Québec Santé during a portion of the time this work was performed.

Institutional Review Board Statement: The study was conducted according to the guidelines of the Declaration of Helsinki and approved by the Research Ethics Board of the Research Institute of the McGill University Health Centre (study code 10-339-PED, 28-03-2011).

Informed Consent Statement: Patient consent was waived due to the nature of the study; all data obtained were administrative and retrospective, containing no identifying information.

Data Availability Statement: The data presented in this study are available on reasonable request from the corresponding author.

Acknowledgments: We thank Vedran Cockovski for assistance in editing, formatting, and proofreading the manuscript and the figures.

Conflicts of Interest: Marc Dorais is self-employed and created his company as a consultant in biostatistics. He has no conflict of interest to report. The other authors also declare no conflict of interest. The funders had no role in the design of the study; in the collection, analyses, or interpretation of data; in the writing of the manuscript, or in the decision to publish the results.

\section{References}

1. Alkandari, O.; Eddington, K.A.; Hyder, A.; Gauvin, F.; Ducruet, T.; Gottesman, R.; Phan, V.; Zappitelli, M. Acute kidney injury is an independent risk factor for pediatric intensive care unit mortality, longer length of stay and prolonged mechanical ventilation in critically ill children: A two-center retrospective cohort study. Crit. Care 2011, 15, R146. [CrossRef]

2. Schaffzin, J.K.; Dodd, C.N.; Nguyen, H.; Schondelmeyer, A.; Campanella, S.; Goldstein, S.L. Administrative data misclassifies and fails to identify nephrotoxin-associated acute kidney injury in hospitalized children. Hosp. Pediatr. 2014, 4, 159-166. [CrossRef] [PubMed]

3. Moffett, B.S.; Goldstein, S.L. Acute kidney injury and increasing nephrotoxic-medication exposure in noncritically-ill children. Clin. J. Am. Soc. Nephrol. 2011, 6, 856-863. [CrossRef]

4. Hui-Stickle, S.; Brewer, E.D.; Goldstein, S.L. Pediatric ARF epidemiology at a tertiary care center from 1999 to 2001. Am. J. Kidney Dis. 2005, 45, 96-101. [CrossRef] [PubMed]

5. Coca, S.G.; Singanamala, S.; Parikh, C.R. Chronic kidney disease after acute kidney injury: A systematic review and meta-analysis. Kidney Int. 2012, 81, 442-448. [CrossRef]

6. Menon, S.; Kirkendall, E.S.; Nguyen, H.; Goldstein, S.L. Acute kidney injury associated with high nephrotoxic medication exposure leads to chronic kidney disease after 6 months. J. Pediatr. 2014, 165, 522-527.e2. [CrossRef]

7. Gansevoort, R.T.; Correa-Rotter, R.; Hemmelgarn, B.R.; Jafar, T.H.; Heerspink, H.J.; Mann, J.F.; Matsushita, K.; Wen, C.P. Chronic kidney disease and cardiovascular risk: Epidemiology, mechanisms, and prevention. Lancet 2013, 382, 339-352. [CrossRef]

8. Wright, J.; Hutchison, A. Cardiovascular disease in patients with chronic kidney disease. Vasc. Health Risk Manag. 2009, 5, 713-722.

9. Askenazi, D.J.; Feig, D.I.; Graham, N.M.; Hui-Stickle, S.; Goldstein, S.L. 3-5 year longitudinal follow-up of pediatric patients after acute renal failure. Kidney Int. 2006, 69, 184-189. [CrossRef] [PubMed]

10. Lefebvre, C.E.; Filion, K.B.; Reynier, P.; Platt, R.W.; Zappitelli, M. Primary Care Prescriptions of Potentially Nephrotoxic Medications in Children with CKD. Clin. J. Am. Soc. Nephrol. 2020, 15, 61-68. [CrossRef]

11. Hessey, E.; Ali, R.; Dorais, M.; Morissette, G.; Pizzi, M.; Rink, N.; Jouvet, P.; Lacroix, J.; Phan, V.; Zappitelli, M. Evaluation of height-dependent and height-independent methods of estimating baseline serum creatinine in critically ill children. Pediatr. Nephrol. 2017. [CrossRef] [PubMed]

12. D'Arienzo, D.; Hessey, E.; Ali, R.; Perreault, S.; Samuel, S.; Roy, L.; Lacroix, J.; Jouvet, P.; Morissette, G.; Dorais, M.; et al. A Validation Study of Administrative Health Care Data to Detect Acute Kidney Injury in the Pediatric Intensive Care Unit. Can. J. Kidney Health Dis. 2019, 6. [CrossRef]

13. Pollack, M.M.; Ruttimann, U.E.; Getson, P.R. Pediatric risk of mortality (PRISM) score. Crit. Care Med. 1988, 16, 1110-1116. [CrossRef] [PubMed]

14. Pampalon, R.; Hamel, D.; Gamache, P.; Philibert, M.D.; Raymond, G.; Simpson, A. An area-based material and social deprivation index for public health in Quebec and Canada. Can. J. Public Health 2012, 103, S17-S22. [CrossRef] [PubMed]

15. Simon, T.D.; Cawthon, M.L.; Stanford, S.; Popalisky, J.; Lyons, D.; Woodcox, P.; Hood, M.; Chen, A.Y.; Mangione-Smith, R.; Center of Excellence on Quality of Care Measures for Children with Complex Needs Medical Complexity Working Group. Pediatric medical complexity algorithm: A new method to stratify children by medical complexity. Pediatrics 2014, 133, e1647-e1654. [CrossRef] 
16. RAMQ. Tableau AM.09: Nombre de participants1 2 et d'ordonnances, nombre d'ordonnances par participant, coût brut des ordonnances et par participant, coût RAMQ des ordonnances et par participant selon le sexe, le groupe d'âge et la catégorie de personnes assurées. In Régime Public D'assurance Médicaments; Régie de l'Assurance Maladie du Québec: Québec, QC, Canada, 2017.

17. Kidney Disease Improving Global Outcomes (KDIGO) CKD Work Group. KDIGO 2012 Clinical Practice Guideline for the Evaluation and Management of Chronic Kidney Disease. Kidney Int. 2013, 3, 5-14.

18. Schwartz, G.J.; Work, D.F. Measurement and estimation of GFR in children and adolescents. Clin. J. Am. Soc. Nephrol. 2009, 4, 1832-1843. [CrossRef]

19. Piepsz, A.; Tondeur, M.; Ham, H. Revisiting normal (51)Cr-ethylenediaminetetraacetic acid clearance values in children. Eur. J. Nucl. Med. Mol. Imaging 2006, 33, 1477-1482. [CrossRef]

20. Hoste, L.; Dubourg, L.; Selistre, L.; De Souza, V.C.; Ranchin, B.; Hadj-Aissa, A.; Cochat, P.; Martens, F.; Pottel, H. A new equation to estimate the glomerular filtration rate in children, adolescents and young adults. Nephrol. Dial. Transplant. 2014, 29, $1082-1091$. [CrossRef]

21. Rink, N.; Zappitelli, M. Estimation of glomerular filtration rate with and without height: Effect of age and renal function level. Pediatr. Nephrol. 2015, 30, 1327-1336. [CrossRef]

22. Hessey, E.; Morissette, G.; Lacroix, J.; Perreault, S.; Samuel, S.; Dorais, M.; Phan, V.; Jouvet, P.; Lafrance, J.P.; LeLorier, J.; et al. Healthcare Utilization after Acute Kidney Injury in the Pediatric Intensive Care Unit. Clin. J. Am. Soc. Nephrol. 2018, 13, 685-692. [CrossRef] [PubMed]

23. Hessey, E.; Perreault, S.; Dorais, M.; Roy, L.; Zappitelli, M. Acute Kidney Injury in Critically Ill Children and Subsequent Chronic Kidney Disease. Can. J. Kidney Health Dis. 2019, 6, 2054358119880188. [CrossRef] [PubMed]

24. Mehta, R.L.; Awdishu, L.; Davenport, A.; Murray, P.T.; Macedo, E.; Cerda, J.; Chakaravarthi, R.; Holden, A.L.; Goldstein, S.L. Phenotype standardization for drug-induced kidney disease. Kidney Int. 2015, 88, 226-234. [CrossRef]

25. Goldstein, S.L.; Kirkendall, E.; Nguyen, H.; Schaffzin, J.K.; Bucuvalas, J.; Bracke, T.; Seid, M.; Ashby, M.; Foertmeyer, N.; Brunner, L.; et al. Electronic health record identification of nephrotoxin exposure and associated acute kidney injury. Pediatrics 2013, 132, e756-e767. [CrossRef]

26. Ingrasciotta, Y.; Sultana, J.; Giorgianni, F.; Caputi, A.P.; Arcoraci, V.; Tari, D.U.; Linguiti, C.; Perrotta, M.; Nucita, A.; Pellegrini, F.; et al. The burden of nephrotoxic drug prescriptions in patients with chronic kidney disease: A retrospective population-based study in Southern Italy. PLoS ONE 2014, 9, e89072. [CrossRef] [PubMed]

27. Goldstein, S.L.; Jaber, B.L.; Faubel, S.; Chawla, L.S.; Acute Kidney Injury Advisory Group of the American Society of Nephrology. AKI transition of care: A potential opportunity to detect and prevent CKD. Clin. J. Am. Soc. Nephrol. 2013, 8, 476-483. [CrossRef] [PubMed]

28. Goldstein, S.L.; Mottes, T.; Simpson, K.; Barclay, C.; Muething, S.; Haslam, D.B.; Kirkendall, E.S. A sustained quality improvement program reduces nephrotoxic medication-associated acute kidney injury. Kidney Int. 2016, 90, 212-221. [CrossRef] [PubMed] 